\title{
Comb multi-wavelength, rectangular pulse, passively mode-locked fiber laser enhanced by un-pumped Erbium-doped fiber
}

\author{
Chunyu Guo ${ }^{\mathrm{a}}$, Ruoheng Luo ${ }^{\mathrm{a}}$, Weiqi Liu ${ }^{\mathrm{a}}$, Shuangchen Ruan ${ }^{\mathrm{a}, *}$, Jinhui Yang ${ }^{\mathrm{a}}$, Peiguang Yan ${ }^{\mathrm{a}}$, Jinzhang \\ Wang $^{\mathrm{a}}$, and Ping Hua ${ }^{\mathrm{b}}$ \\ a Shenzhen Key Laboratory of Laser Engineering, Key Laboratory of Advanced Optical Precision Manufacturing Technology of Guangdong \\ Higher Education Institutes, College of Optoelectronic Engineering, College of Electronic Science and Technology, Shenzhen University, \\ Shenzhen, 518060, China \\ b Optoelectronics Research Centre, University of Southampton, SO171BJ, UK
}

\begin{abstract}
We propose and demonstrate a comb multi-wavelength, nanosecond rectangular pulse, passively mode-locked Erbium-doped fiber (EDF) laser. A section of un-pumped EDF had been employed to optimize the multi-wavelength pulses for the first time to the best of our knowledge. The un-pumped EDF absorbs the unwanted the short-wavelength lasing and optimizes the gain, therefore allowing for the enhancement of the long-wavelength lasing. Because of the gain competition effect in the un-pumped EDF, the output wavelength line number of the fiber laser can be significantly increased from three wavelengths to twenty lasing wavelengths. The mode-locked pulse has a rectangular temporal profile with pump power dependent pulse duration. Experimental results illustrate that the fiber laser has a good stability at room temperature. This work provides a new configuration for the design of multi-wavelength, rectangular nanosecond pulse that may fit for specific applications.
\end{abstract}

Keywords: multi-wavelength fiber laser, Erbium-doped fiber laser, rectangular pulse

\section{Introduction}

Rectangular pulse lasers have attracted significant interest for their wide and important applications in many fields, such as all-optical rectangular-wave clocks, fiber grating, and laser micromachining [1-3]. Recently, dissipative soliton resonance (DSR) was theoretically proposed to achieve wave-breaking-free rectangular pulse by properly choosing parameters in the frame of complex Ginzburg-Landau equation [4-7]. By using the nonlinear polarization rotation (NPR) mode-locked technique, the rectangular pulses have been experimentally investigated in fiber ring lasers $[8,9]$. The rectangular pulse has also been reported in figure-of-eight fiber lasers with net normal dispersion [10] and anomalous dispersion [11]. According to the previous work [8-12], all of the rectangular pulse mode-locked fiber lasers operate in a certain wavelength range. In contrast, little work about multi-wavelength rectangular pulse fiber laser had been reported.

On the other hand, multi-wavelength pulsed fiber lasers have received considerable attention for their versatile applications, including wavelength division multiplexer (WDM) fiber communication systems, photonic component characterization and optical signal processing [13-16]. Recently, Zhang et al. reported a triple-wavelength passively mode-locked fiber laser in an all-normal dispersion cavity with a semiconductor saturable absorber mirror (SESAM) [17]. Luo et al. demonstrated a multi-wavelength fiber laser by using a SESAM and an inline birefringence fiber filter [18]. Up to seventh lasing wavelengths mode-locked pulse in $3 \mathrm{~dB}$ bandwidth with $3.65 \mathrm{~nm}$ channel spacing are obtained. The number of the lasing wavelength is an important performance parameter for the multi-wavelength fiber laser. And a desirable multi-wavelength source needs large channel count when the channel spacing is given. However, most of the previous research about multi-wavelength mode-locked fiber laser focuses only on the generation of multi-wavelength lasing. And no

*Corresponding author.

E-mail addresses: scruan@szu.edu.cn (S.Ruan). 
experimental work has undertaken until now to investigate the improvement on the number of lasing wavelength in a multi-wavelength mode-locked fiber laser.

In this paper, we have experimentally demonstrated a comb multi-wavelength, nanosecond rectangular pulse, passively mode-locked EDF laser by using the nonlinear amplifying loop mirror (NALM) technique and a fiber Sagnac loop comb filter. A section of un-pumped EDF has been inserted in the cavity to operate as a booster which transfers the energy from the higher-energy resonance modes to the lower-energy resonance modes. It is the first time to use the un-pumped EDF for the enhancement of multi-wavelength output in a mode-locked fiber laser. Up to 20 lasing wavelengths are obtained, which represents about six times increase in the number of the generated wavelengths compared with that from the fiber laser without the un-pumped EDF.

\section{Experimental setup}

The experimental setup of the proposed multi-wavelength mode-locked EDF laser is schematically shown in Fig. 1. It is a figure-of-eight configuration which consists of a NALM and a unidirectional ring (UR). A piece of $7 \mathrm{~m}$ EDF (EDF1) with an absorption coefficient of $23.9 \mathrm{~dB} / \mathrm{m} @ 979 \mathrm{~nm}$ is used as the gain medium. It is pumped by a $976 \mathrm{~nm}$ single-mode laser diode (LD) with the maximum output power of $620 \mathrm{~mW}$ through a 980/1550 nm WDM. A segment of $118 \mathrm{~m}$ high-nonlinearity fiber (HNF) with a nonlinear coefficient of $10 / \mathrm{W} / \mathrm{km}$ is introduced into the NALM cavity to increase the intra-cavity nonlinearity. Two polarization controllers (PC1 and PC2) are used to adjust the polarization state of the circulating light. A section of $1.7 \mathrm{~m}$ EDF (EDF2) with the same parameters of EDF1 is inserted in the UR cavity. The fiber optical circulator (CIR) plays the role of an isolator which enforces the unidirectional operation. A fiber Sagnac loop which is made up of a $3 \mathrm{~dB}$ optical coupler (OC), PC3 and $4 \mathrm{~m}$ polarization maintaining fiber (PMF), is used as a comb filter to select the lasing channels. The output power is extracted from the cavity through a $10 / 90$ OC. A $1 \mathrm{GHz}$ digital phosphor oscilloscope (Tektronix DPO7014C) together with a $45 \mathrm{GHz}$ high-speed photoelectric detector (New Focus 1014) is employed to monitor the traces of the mode-locked pulses. An optical spectrum analyzer (OSA, YOKOGAWA AQ6370B) is used to measure the optical spectrum.

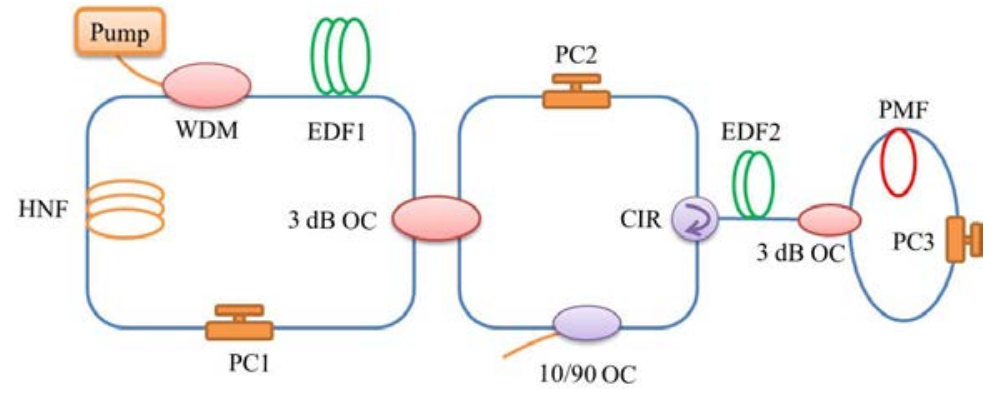

Fig.1 Experimental setup of the multi-wavelength mode-locked fiber laser. EDF: erbium-doped fiber; WDM: wavelength division multiplexer; HNF: high nonlinearity fiber; PMF: polarization maintaining fiber; PC: polarization controller; OC: optical coupler; CIR: circulator.

\section{Result and discussion}

The NALM technique is employed to realize the passively mode-locked state in our fiber laser. By performing the comparison experiment on multi-wavelength fiber laser with or without using EDF2, we are able to understand the contribution of the un-pumped EDF.Firstly, we conduct the experiment without using EDF2. By properly adjusting the orientation of the PCs, the self-started multi-wavelength rectangular pulses mode-locked state can be achieved when the pump power exceeds the threshold. Figure 2 shows a typical mode-locked state of the multi-wavelength fiber laser at the pump power of $560 \mathrm{~mW}$. Figure 2 (a) is the oscilloscope trace of the output pulse with the repetition rate of $1.47 \mathrm{MHz}$. The mode-locked pulse train has a uniform pulse interval of about $680.1 \mathrm{~ns}$ which corresponds well to the cavity roundtrip time. The inset of Fig. 2 (a) presents the profile of a single rectangular pulse with the pulse width of 18.87 ns. Figure 2 (b) shows the corresponding optical spectrum of the rectangular pulse. Three lasing wavelengths within $3 \mathrm{~dB}$ bandwidth (3.46 nm) are obtained with the lasing location centered at $1568.06 \mathrm{~nm}$. However, there are up to 21 lasing wavelengths within $10 \mathrm{~dB}$ bandwidth. Thus, if the lasing wavelengths around $1568 \mathrm{~nm}$ can be suppressed, the output spectrum will become more flat and the number of output wavelength within $3 \mathrm{~dB}$ bandwidth will be significantly increased. 

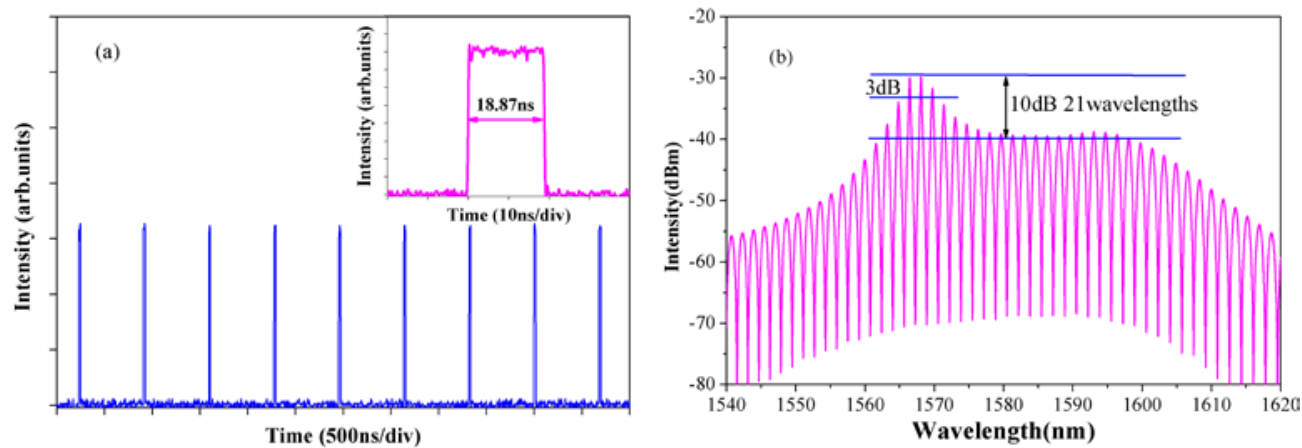

Fig. 2 Typical mode-locked state without EDF2. (a) Oscilloscope trace of the rectangular pulse. Inset: a single pulse. (b) Corresponding optical spectrum.

In the following experiment, a $1.4 \mathrm{~m}$-long EDF was inserted into the laser system. In the temporal domain, the mode-locked pulse shown on the oscillograph is almost the same as that without EDF2 except that the repetition rate $(1.434 \mathrm{MHz}$ ) is slightly different. However, the output spectrum is more flat as shown in Fig. 3 (a). There are up to 20 lasing wavelengths within $3 \mathrm{~dB}$ bandwidth $(31.91 \mathrm{~nm})$, with 26 lasing wavelengths within $10 \mathrm{~dB}$ bandwidth. The signal-to-noise (SNR) is about $28 \mathrm{~dB}$. The efficiency improvement on the number of lasing wavelength is attributed to the utilization of un-pumped EDF2 section. In the experiment, the un-pumped EDF2 absorbs the unwanted short-wavelength energy of the circulating light as a secondary pump source to enhance the strength of the long-wavelength lines. Since the lasing gain is clamped at the cavity loss in a running laser, the un-pumped EDF2 was also used for optimizing the gain of the NALM and introducing additional cavity loss to get a uniform spectrum. Since the lasing gain is clamped at the cavity loss in a running laser, the un-pumped EDF2 was also used for optimizing the gain of the NALM and introducing additional cavity loss to get a uniform spectrum [19]. Thus, the output spectrum gets more flat and the lasing wavelength count within $3 \mathrm{~dB}$ bandwidth is increased. Figure 3 (b) is the enlarged spectrum range from $1588 \mathrm{~nm}$ to $1602 \mathrm{~nm}$. As can be seen, the wavelength spacing is $1.67 \mathrm{~nm}$, coinciding with the free spectral range of the comb filter from the formula $\Delta \lambda=\lambda^{2} /(B \times L)$, where $\lambda$ is the operation lasing wavelength, B and L are the birefringence coefficient and the length of the PMF.
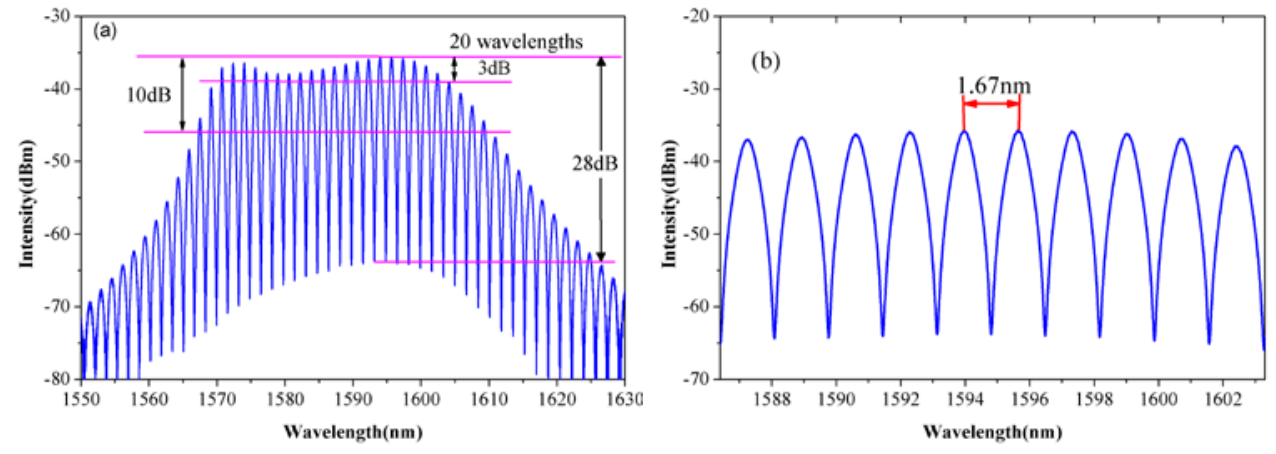

Fig. 3 (a) The output spectrum optimized by EDF2; (b) The enlarged output spectrum.

The stability of the multi-wavelength passively mode-locked fiber laser is essential for the practical applications. To verify the lasing wavelength stability of the fiber laser, we repeatedly scanned the multi-wavelength output 10 times in one hour with an interval of 6 minutes. The measurement results of the main 20 lasing wavelengths are presented in Fig. 4. No significant wavelength drift was observed. For better clarity, Figure 5 shows the power variations of four channels in the experimental observation. The lasing wavelengths are $1572.384 \mathrm{~nm}, 1580.568 \mathrm{~nm}, 1588.936 \mathrm{~nm}$, and $1597.308 \mathrm{~nm}$, corresponding to channel 2, channel 7, channel 12, and channel 17, respectively. The maximum power fluctuations of the selected channels are $0.291 \mathrm{~dB}, 0.15 \mathrm{~dB}, 0.11 \mathrm{~dB}$, and $0.103 \mathrm{~dB}$, respectively. All of them are lower than $0.3 \mathrm{~dB}$. These results indicate that the proposed multi-wavelength passively mode-locked fiber laser can operate stably at room temperature. 


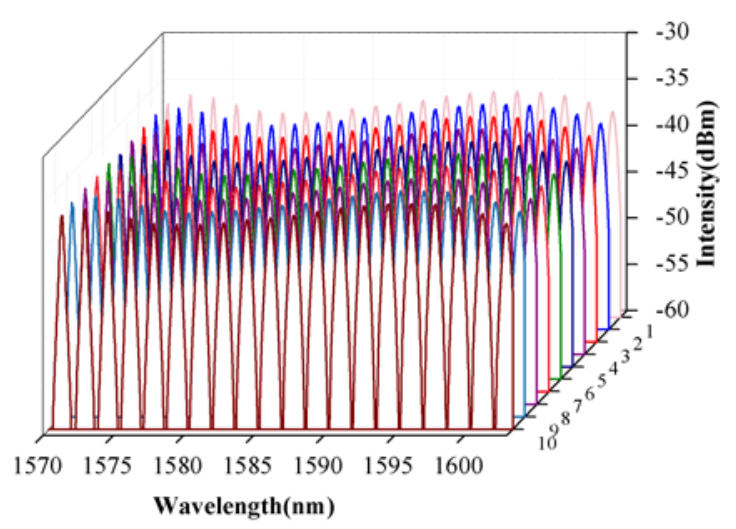

Fig. 4 Repeatedly scanned output 10 times with a 6 min interval.

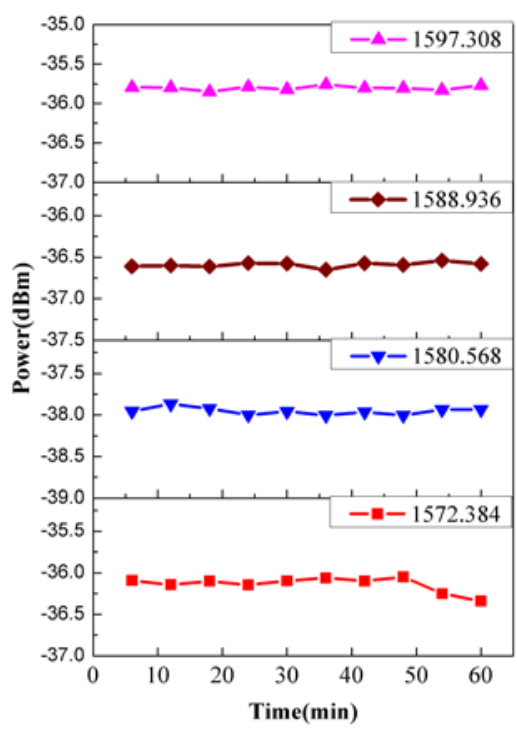

Fig. 5 Power fluctuation of four channels.

By fixing the PCs, the fiber laser can maintain the stable multi-wavelength oscillation at different pump power. The spectra under different pump power are presented in Fig. 6 . The spectral intensity obviously increases when we adjust the pump power from $290 \mathrm{~mW}$ to $620 \mathrm{~mW}$, and the $3 \mathrm{~dB}$ bandwidth slightly increases with the increase of pump power. The maximum $3 \mathrm{~dB}$ bandwidth fluctuations is $0.15 \mathrm{~nm}(31.82 \mathrm{~nm}-31.97 \mathrm{~nm}$ ) which is far smaller than the $3 \mathrm{~dB}$ bandwidth and the wavelength spacing. Thus, the fiber laser keeps the 20 lasing wavelengths output, and the location of lasing wavelengths almost maintains the same positions relative to each other.

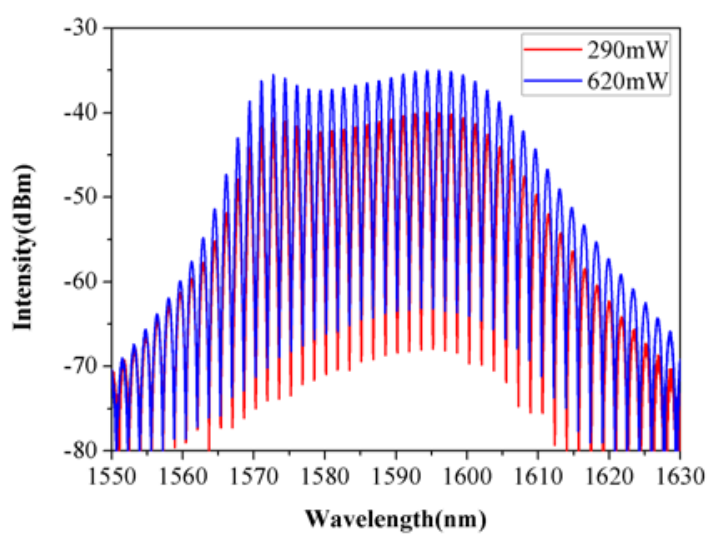

Fig. 6 Optical spectra under different pump powers.

Moreover, the pulse characteristics have also been experimentally investigated. We find that the pulse width of the output 
rectangular pulse can be tuned by adjusting the pump power despite that the PCs are fixed. The dynamic of the rectangular pulse broadening with the increasing pump power is depicted in Fig. 7 (a). As can be seen, the pulse width broadens with the increase of pump power, while the pulse amplitude almost keeps invariable during the process of pulse broadening. This is in good agreement with the principles of DSR, which shows that things fit together well. It is worth noting that no fine structure or multi-pulse oscillation was observed during the pulse broadening process. And the rectangular pulse is still stable at the maximum pump power of $620 \mathrm{~mW}$. Figure 7 (b) shows the evolution of the pulse width and the output power versus pump power. Both of them monotonously increase with the increase of pump power. The pulse width broadens from $5.49 \mathrm{~ns}$ to $20.88 \mathrm{~ns}$ when the pump power is increased from $265 \mathrm{~mW}$ to $620 \mathrm{~mW}$. At a maximum pump power of $620 \mathrm{~mW}$, the largest output power is $0.81 \mathrm{~mW}$. And there appears no output power saturation phenomenon. Considering the repetition rate of $1.434 \mathrm{MHz}$, the output pulse energy is estimated as $0.56 \mathrm{~nJ}$.
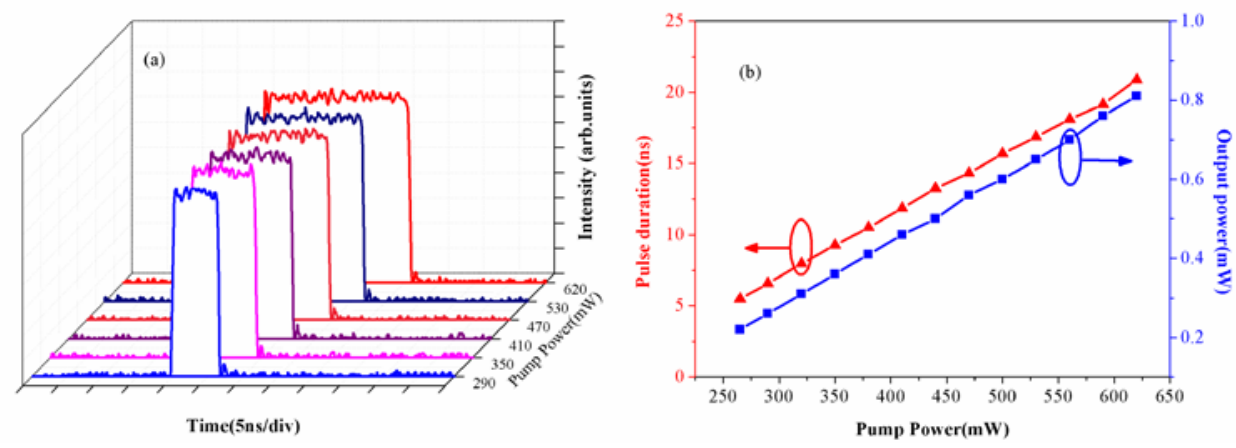

Fig. 7 (a) Rectangular pulses under different pump powers. (b) Pulse widths and output powers versus the pump powers.

To better show the quality of the mode-locked pulses, we have measured the radio frequency (RF) spectrum by a RF spectrum analyzer with the bandwidth of $3 \mathrm{GHz}$ (Agilent N9302A). The RF spectra of the mode-locked pulses around the fundamental and harmonic repetition rates are shown in Fig.8. The resolution bandwidths are $300 \mathrm{~Hz}$ and $10 \mathrm{KHz}$, respectively. The fundamental frequency peak is located at $1.434 \mathrm{MHz}$ and the SNR is about $67 \mathrm{~dB}$. The inset of Fig.8 performs the wideband RF spectrum up to $200 \mathrm{MHz}$. The results demonstrate that the fiber laser operates at a stable mode-locked sate.

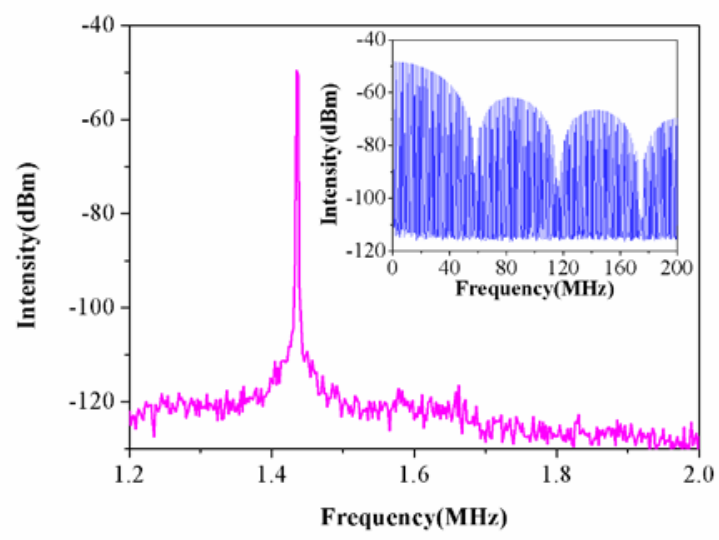

Fig.8. RF spectrum of the output pulses. Inset: wideband RF spectrum up to $200 \mathrm{MHz}$.

\section{Conclusion}

In conclusion, a comb multi-wavelength, nanosecond rectangular pulse, passively mode-locked EDF laser has been experimentally demonstrated by using the NALM technique and a Sagnac loop filter. A section of un-pumped EDF has been utilized to enhance the lasing wavelength emission. And the output wavelength of the fiber laser is significantly increased from 3 lasing wavelengths to 20 lasing wavelengths within a $3 \mathrm{~dB}$ bandwidth. The channel spacing is $1.67 \mathrm{~nm}$ and the SNR is $28 \mathrm{~dB}$. The pulse width broadens from $5.49 \mathrm{~ns}$ to $20.88 \mathrm{~ns}$ when the pump power is increased from $265 \mathrm{~mW}$ to $620 \mathrm{~mW}$. The multi-wavelength passively mode-locked fiber laser operates stably at room temperature. Our work provides a new solution for the improvement on the number of lasing wavelength in a multi-wavelength mode-locked fiber laser. More 
importantly, it is anticipated that our work may not only benefit for the development of multi-wavelength mode-locking by the SESAM technique, but also gives some new insights for the development of other types of the multi-wavelength mode-locking fiber lasers by using the emerging new types of saturable absorbers, from graphene [20], topological insulator [21] to Molybdenum Disulfide $\left(\mathrm{MoS}_{2}\right)$ saturable absorber [22]. In the future, the multi-wavelength lasing by the mentioned new saturable absorbers may debut.

\section{Acknowledgement}

This work was supported by National Natural Science Foundation of China (NSFC) (61308049); National High-tech R\&D Program of China (863 Program) (2015AA021102); Outstanding Young Teacher Cultivation Projects in Guangdong Province (YQ2015142); Shenzhen Science and Technology Project (JCYJ20160520161351540 \& JCYJ 20160427105041864).

\section{References}

[1]. M. A. Putnam, M. L. Dennis, I. N. Duling III, C. G. Askins, E. J. Friebele, Broadband square-pulse operation of a passively mode-locked fiber laser for fiber Bragg grating interrogation, Opt. Lett. 23 (1988) 138-140.

[2]. X. Peng, B. Jordens, A. Hooper, B. W. Baird, W. Ren, L. Xu, L. Sun, Generation of programmable temporal pulse shape and applications in micromachining, Proc. of SPIE 7193 (2009) 719324.

[3]. A. M. Kaplan, G. P. Agrawal, D. N. Maywar, Optical square-wave clock generation based on an all-optical flip-flop, IEEE Photon. Technol. Lett. 22 (2010) 489-491.

[4]. W. Chang, A. Ankiewicz, J. M. Soto-Crespo, N. Akhmediev, Dissipative soliton resonances, Phys. Rev. A 78 (2008) 23830.

[5]. W. Chang, J. M. Soto-Crespo, A. Ankiewicz, N. Akhmediev, Dissipative soliton resonances in the anomalous dispersion regime, Phys. Rev. A 79 (2009) 33840.

[6]. N. Akhmediev, J. M. Soto-Crespo, P. Grelu, Roadmap to ultra-short record high-energy pulses out of laser oscillators, Phys. Lett. A 372 (2008) 3124-3128.

[7]. P. Grelu, W. Chang, A. Ankiewicz, J. M. Soto-Crespo, N. Akhmediev, Dissipative soliton resonance as a guideline for high-energy pulse laser oscillators, J. Opt. Soc. Am. B 27 (2010) 2336-2341.

[8]. X. Wu, D. Y. Tang, H. Zhang, L. M. Zhao, Dissipative soliton resonance in an all-normal-dispersion erbium-doped fiber laser, Opt. Express 17 (2009) 5580-5584.

[9]. L. Duan, X. Liu, D. Mao, L. Wang, G. Wang, Experimental observation of dissipative soliton resonance in an anomalous-dispersion fiber laser, Opt. Express 20 (2012) 265-270.

[10].J. Yang, C. Guo, S. Ruan, D. Ouyang, H. Lin, Y. Wu, R. Wen, Observation of Dissipative Soliton Resonance in a Net-Normal Dispersion Figure-of-Eight Fiber Laser, IEEE Photon. J. 5 (2013) 1500806.

[11].S. Wang, Q. Ning, A. Luo, Z. Lin, Z. Luo, W. Xu, Dissipative soliton resonance in a passively mode-locked figure-eight fiber laser, Opt. Express 21 (2013) 2402-2407.

[12]. J. Lee, J. Koo, and J. H. Lee, A pulse-width-tunable, mode-locked fiber laser based on dissipative soliton resonance using a bulk-structured $\mathrm{Bi}_{2} \mathrm{Te}_{3}$ topological insulator, Opt. Eng. 55 (2016) 081309

[13].Y. Wang, L. Xia, C. Yang, Y. Zhang, L. Li, Z. Xie, S. Fu, D. Liu, Multiwavelength generation based on a mode-locked fiber laser using carbon nanotube and fiber Fabry-Perot filter, Appl. Optics 52 (2013), 6616-6619.

[14].Z. T. Wang, Y. Chen, C. J. Zhao, H. Zhang, S. C. Wen, Switchable dual-wavelength synchronously Q-switched erbium-doped fiber laser based on graphene saturable absorber, IEEE Photon. J. 4 (2012) 869-876. 
[15].Z. Luo, A. Luo, W. Xu, Multiwavelength picosecond and single wavelength femtosecond pulses emission in a passively mode-locked fiber laser using a semiconductor saturable absorber mirror and a contrast ratio tunable comb filter, Appl. Optics 50 (2011) 2831-2835.

[16]. Y. Chen, M. Wu, P. Tang, S. Chen, J. Du, G. Jiang, Y. Li, C. Zhao, H. Zhang, and S. Wen, The formation of various multi-soliton patterns and noise-like pulse in a fiber laser passively mode-locked by a topological insulator based saturable absorber, Laser Phys. Lett. 11 (2014) 055101

[17].H. Zhang, D. Y. Tang, X. Wu, L. M. Zhao, Multi-wavelength dissipative soliton operation of an erbium-doped fiber laser, Opt. Express 17 (2009) 12692-12697.

[18].Z. Luo, A. Luo, W. Xu, Tunable and switchable multiwavelength passively mode-locked fiber laser based on SESAM and inline birefringence comb filter, IEEE Photon. J. 3 (2011) 64-70.

[19]. X. Liu, L. Zhan, S. Luo, Z. Gu, J. Liu, Y. Wang, and Q. Shen, Multiwavelength erbium-doped fiber laser based on a nonlinear amplifying loop mirror assisted by un-pumped EDF, Opt. Express 20 (2012) 7088-7094

[20].H. Zhang, D. Tang, R. J. Knize, L. Zhao, Q. Bao, K. P. Loh, Graphene mode-locked, wavelength-tunable, dissipative soliton fibre laser, Appl. Phys. Lett. 96 (2010) 111112.

[21].S. Chen, C. Zhao, Y. Li, H. Huang, S. Lu, H. Zhang, S. Wen, Broadband optical and microwave nonlinear response in topological insulator, Opt. Mater. Express 4 (2014) 587-596.

[22].H. Zhang, S. B. Lu, J. Zheng, J. Du, S. C. Wen, D. Y. Tang, K. P. Loh, Molybdenum disulfide $\left(\mathrm{MoS}_{2}\right)$ as a broadband saturable absorber for ultra-fast photonics, Opt. Express 22 (2014) 7249-7260. 